\section{Adjunktive Therapieoptionen bei schweren bakteriellen Infektionen}

\author{
N. Suttorp ${ }^{1}$ \\ ${ }^{1}$ Med. Klinik-Infektiologie und Pneumologie, Charité, \\ Universitätsmedizin Berlin
}

Auch wenn Antiinfektiva sachgerecht gegeben in kurzer Zeit alle Pathogene abtöten, kann der Patienten trotzdem sterben. Ursache kann das Ausmaß der bisher schon eingetretenen Schäden sein. Auch kommt eine immunologische Überreaktion mit konsekutiver (weiterer) Gewebedestruktion infrage. Bei dieser Betrachtung werden bisher in diesem Zusammenhang wenig beleuchtete Vorgänge und deren potenzielle Beeinflussung wichtig - wie Reduktion erhöhter Gefäßpermeabilität, Förderungen der Auflösung einer Entzündungsreaktion (resolution) und der Geweberegeneration, Vermeidung einer systemischen Ausbreitung einer initial lokalen Infektion (containment), Förderung der Apoptose proinflammatorischer und Vermeidung der Apoptose von strukturellen Zellen. Alle diese Vorgänge können durch eine am Wirt orientierte adjunktive Therapie günstig beeinflusst werden.

Darüber hinaus ist eine am Pathogen orientierte adjunktive Therapie machbar. Zum Beispiel können Produkte von Bakteriophagen Bakterien wirksam abtöten, ohne dass ein Resistenzdruck, wie er durch Antibiotika erzeugt wird, entsteht.

Drei Beispiele für eine am Wirt orientierte adjunktive Therapie seien genannt:

I) Eine erhöhte Gefäßpermeabilität ist ein Kardinalsymptom der Entzündung. Sowohl die para- wie auch der transendotheliale Flüssigkeitsverschiebungen sind massiv gesteigert. Die zugrunde liegenden Mechanismen (Abbau interendothelialer Junktionen, vermehrte Endothelzellkontraktion) sind eingehend charakterisiert [1]. Untersuchungen haben gezeigt, dass z. B. Adrenomedullin oder Angiopoetin-1 erhöhte Permeabilität wirksam reduzieren können [2 -4]. Eine ähnliche Wirkung auf die Gefäßpermebailität kann durch Hemmung von bestimmten Isoenzymen der Phosphodiesterasegruppe erreicht werden [5 - 7].

II) Protektine und Resolvine als Produkte des Lipoxygenasewegs fördern die Resolution und sind sehr geeignet, Heilung zu beschleunigen [8].

III) Bronchoskopisch platziertes GM-CSF kann bei akuter schwerer Lungenschädigung die Oxygenierung verbessern [9].
Ein Beispiel einer am Pathogen orientierten adjunktiven Therapie sei genannt:

Das Bakteriophagenprodukt Endolysin Cpl-1 ist, wenn es in therapeutischer Absicht inhalativ oder intraperitoneal gegeben wird, in der Behandlung einer Pneumokokkenpneumonie so wirksam wie Amoxicillin [10,11].

Danksagung: Eigene Arbeiten wurden durch das BMBF (CAPNETZ, PROGRESS, CAPSyS) sowie durch die DFG (SFB-TR84) unterstützt.

Interessenkonflikte: Es bestehen keine Interessenkonflikte.

\section{Literatur}

1 Müller-Redetzky HC, Suttorp N, Witzenrath M. Dynamics of pulmonary endothelial barrier function in acute inflammation: mechanisms and therapeutic perspectives. Cell Tissue Res 2014; 355: 657-673

2 Hippenstiel SM, Witzenrath B, Schmeck AC et al. Adrenomedullin reduces endothelial hyperpermeability. Circ Res 2002; 91: 618-625

3 Temmesfeld-Wollbrück B, Hocke AC, Suttorp $N$ et al. Adrenomedullin and endothelial barrier function. Thromb Haemost 2007; 98: 944-951

4 Müller HC, Witzenrath M, Tschernig T et al. Adrenomedullin attenuates ventilator-induced lung injury in mice. Thorax 2010; 65: 1077-1084

5 Suttorp N, Weber $U$, Welsch T et al. Role of phosphodiesterases in the regulation of endothelial permeability in vitro. J Clin Invest 1993; 91: $1421-1428$

6 Seybold J, Thomas D, Witzenrath $M$ et al. Tumor necrosis factor-dependent expression of phosphodiesterase 2: role in endothelial hyperpermeability. Blood 2005; 105: 3569-3576

7 Suttorp N, Fuhrmann M, Tannert-Otto S et al. Pore forming bacterial toxins potently induce release of nitric oxide in porcine endothelial cells. J Exp Med 1993; 178: 337-341

8 Levy BD, Serhan $C N$. Resolution of acute inflammation in the lung. Annu Rev Physiol 2014; 76: 467-492

9 Herold S, Hoegner K, Vadász I et al. Inhaled granulocyte/macrophage colony-stimulating factor as treatment of pneumonia-associated acute respiratory distress syndrome. Am J Respir Crit Care Med 2014; 189: 609-611

10 Doehn JM, Fischer K, Reppe K. Delivery of the endolysin Cpl-1 by inhalation rescues mice with fatal pneumococcal pneumonia. J Antimicrob Chemother 2013; 68: 2111-2117

11 Witzenrath M, Schmeck B, Doehn JM et al. Systemic use of the endolysin Cpl-1 rescues mice with fatal pneumococcal pneumonia. Crit Care Med 2009; 37: $642-649$

Bibliografie

DOI http://dx.doi.org/10.1055/s-0033-1358032

Drug Res 2014; 64, Suppl. 1: S13-S13

(c) Georg Thieme Verlag KG Stuttgart · New York .

ISSN 2194-9379

Korrespondenzadresse

Norbert Suttorp

Charité - Campus Mitte

Charitéplatz 1

10117 Berlin

norbert.suttorp@charite.de 